Submitted to the 1987 Synchrotron Radiation Instrumentation Conference, Madison, Wiscionsin, June 2l-25, 1987

$$
\text { CONF-870 } 6010-8
$$

BNL 39982

\title{
A NEW APPARATUS FOR THE STUDY OF NUCLEAR BRAGG SCATTERING
}

\author{
D. P. Siddons, J. B. Hastings and G. Faigel \\ National Synchrotron Light Source \\ Brookhaven National Laboralory \\ Upton, Neu' York 11978, U.S.A.
}

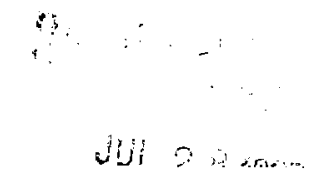

BNL --39982

DE87 012481

\begin{abstract}
A new monochromator system has been constructed which provides an energy resolution of $0.005 \mathrm{eV}$ and an angular divergence of 0.4 arc seconds at an energy of $14.413 \mathrm{keV}$. In conjunction with a highly perfect erystal of isotopically enriched ${ }^{57} \mathrm{Fe}_{2} \mathrm{O}_{3}$. a beam of nuclear resonant photons was extracted from the synchrotron continuum with signal to noise ratio of $100: 1$, and an intensity of $>2$ quanta/sec.
\end{abstract}

PACS Vumbers 76.80.-Y. 07.85.-T. 42.80.Dk. $7 \pm .90 .+1$

\section{DISCLAIMER}

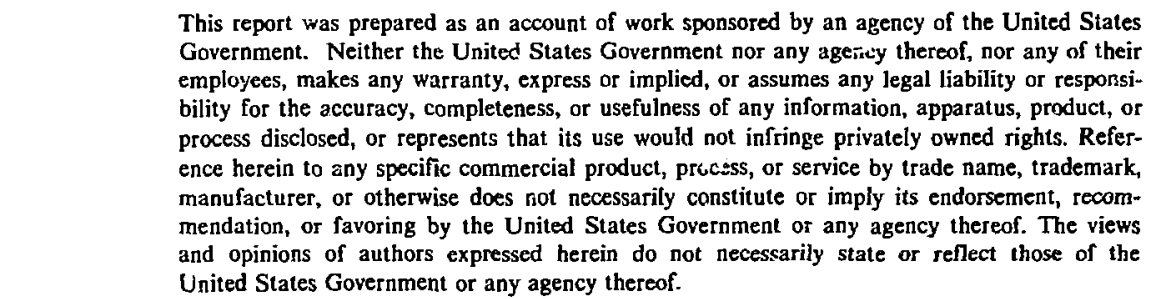

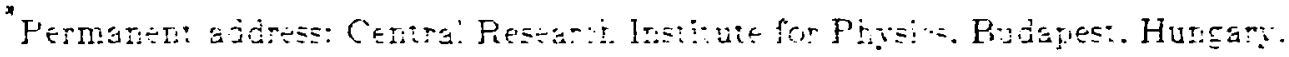




\section{Introduction}

The work of Mössbauer [1] showed that radiation having a very narrow energy width was produced by the recoil-free decay of nuclear excited states in solids. The nuclear excited states are produced as one product of a radioactive decay, and so only those nuclear species which are the product of a suitable decay scheme can be studied. The ready availability of highly intense $x$-ray beams from sypchrotron sources in recent years has raised the possibility of directly exciting the resonance by nuclear photoabsorption [2]. The probability of absorption (and subsequent re-emission) is negligible except for photon energies very close to the resonance energy. and in the case of ${ }^{5 i} F \boldsymbol{c}$. this is at $14.413 \mathrm{kel}$. in a handwidtb of about $10^{-9} \mathrm{el}$. The problen! of deteting the small number of photons within this bandwidth when using synchrotron radiation to excite such resozarces is thus essentially one of signal to noise ratic.

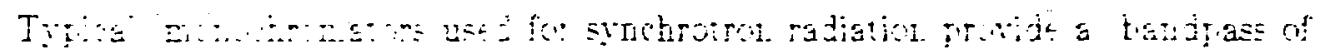

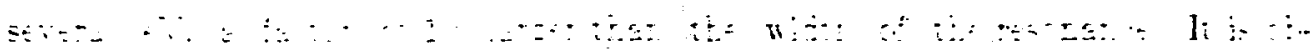

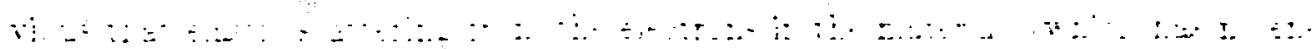

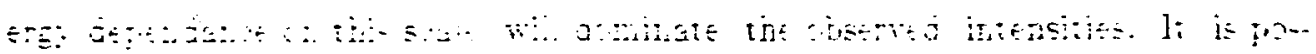

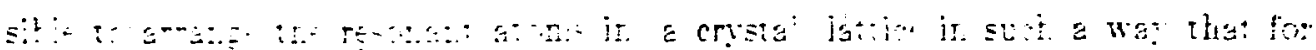

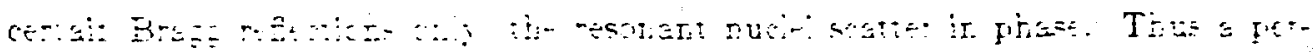

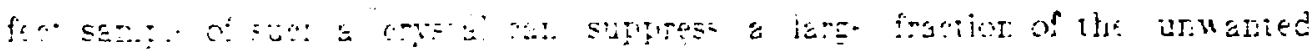

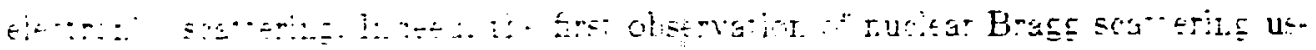

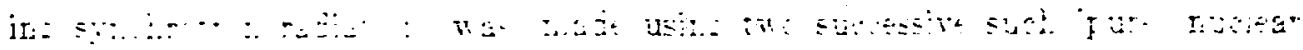


reflections [3]. Even so, the signal to noise ratio achieved was only around unity. The number of systems for which two perfect isotopically enriched crystals are available is severely limited. and details of the resulting photon beam, whilst intrinsically interesting, are greatly complicated by the double nuclear scattering. We were thus prompted to try to develop an approach which might allow obseryation of the nuclear scattering in cases other than the magnetic systems used to date, in particular for situations where the electronic scattering is less perfectly suppressed. and to extend the capabilities to nuclei other than iron.

\section{High Resolution Monochromators.}

\subsection{Resolution}

The choice of a basic design for a high-resolution $x$-ray monochromator

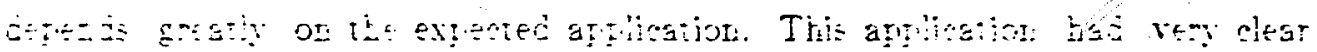

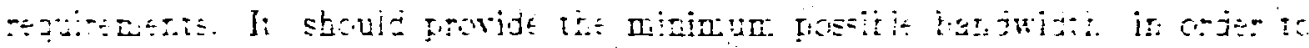

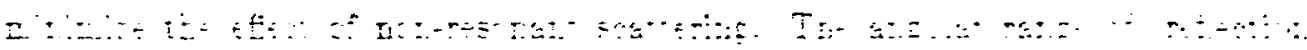

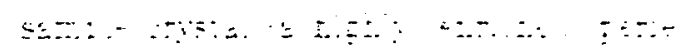

й i s s- at a

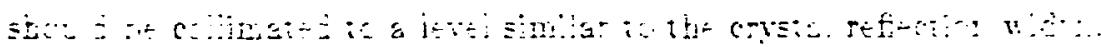

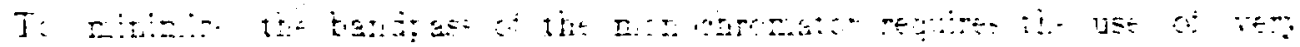

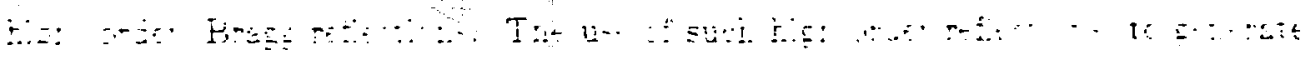


extremely narrow wavelength bands from sychrotron source has been the subject of several investigations with the aim of observing inelastic $x$-ray scattering from pbonons and other sub-el excitations [4.5.6]. Those studies were able to choose freely the diffaction order utilized. since their choice of photon energy was arbitrary. The experiment by Graeff and Materlik [4] demonstrated that resolutions of millivolt order could in fact be observed. Their apparatus gave a resolution of $0.008 \mathrm{el}^{-}$at an energy of $15.8 \mathrm{kel}^{-}$using the silicon $(8,8,8)$ reflection. The study of inelastic $x$-ray scattering. bowever. requires the collection of large solid angles of radiation due to the diffuse and extremely weak nature of the scattering cross-sections. Consequently. a practical apparatus requires the use of focussins optics. as in the work of Dorner. Burkel and Peisl [5]. and that of Siddons et al. [6;. The type of focussins ortics used in those experiments would noi be benefeial to the present experizent. since ther would provide a divergeri excitation beam. As fointed out abste. on!y rays incident

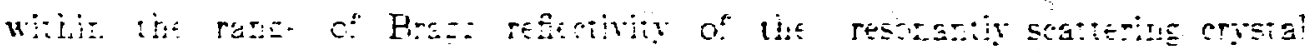

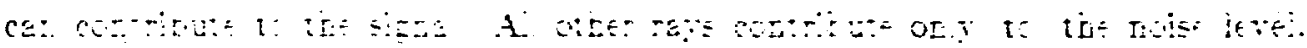

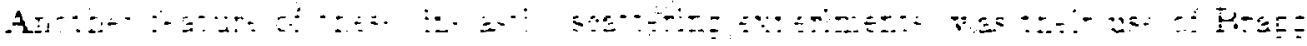

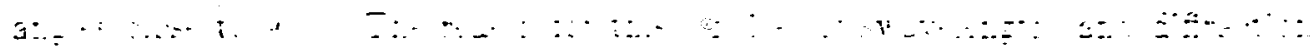

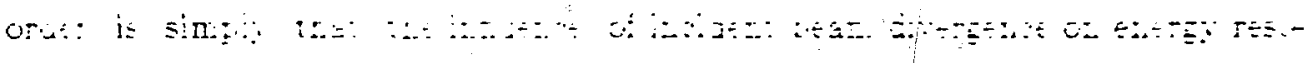

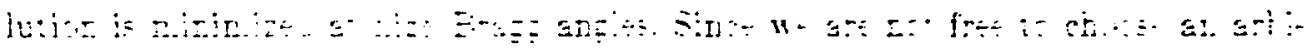

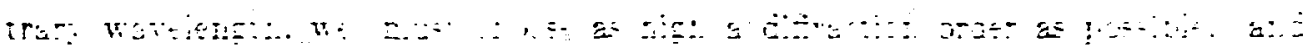
ce

It. $\quad \therefore \quad \therefore \quad \therefore$ 
double-crystal arrangement. In its classic configuration [i] it is somewhat unwieldy, since the monochromatic beam is deviated from the primary beam by four times the Bragg angle. A system in which the output beam is fixed in space (or at least in direction) is significantly easier to instrument. As pointed out by Beaumont and Hart [8], the availability of highly perfect crystals of silicon in large pieces makes possible the design of many-reflection systems with little cost in efficiency. A realization of the dispersive double-cristal apparatus using a pair of 2-reflection monoliths provides a fxed exit tunable beam of wellcontrolled divergence. Its resolution is essentially independant of the source characteristics. The highest order reflection arailable for diffraction at $14.413 \mathrm{keV}^{-}$ by silicon is the (11.5.3). For practical reasons we chose not to use this. but the (10.6.4). Tot only is it easier to fabricate the tworeflection monoliths for this reflection. but reflections of even order have a better peak reflectivity. The bandwidth frovided by this combination wa nosel. with an angular dive:-

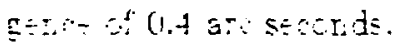

2.2. Triernai considerations

$$
\begin{aligned}
& \text { a- Xs: }
\end{aligned}
$$

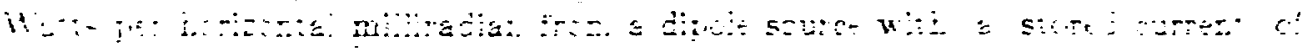

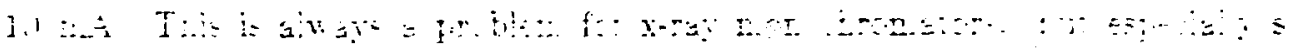

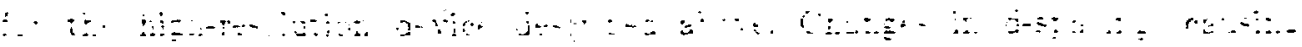

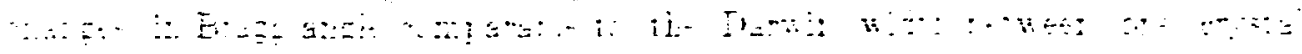

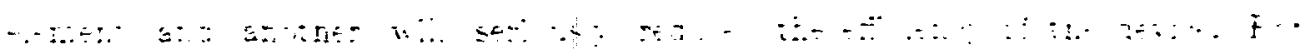


the $(10,6,4)$ reflection at $14.413 \mathrm{keV}$, such changes in d-spacing occur for temperature differentials of roughly $0.2 \%$. With a power load of 8 Wat ts incident on one wafer of a 2-reflection monolith. it is quite difficult to maintain the temperature differential across the device to this level. Even though such changes can be compensated by suitable crystal design, time variations in the power load would render control unwieldy. It is also vital that the absolute energy of the transmitted beam remain constant (i.e. on resonance). and that is far more difficult to achieve if the temperature of the crystal elements is poorly defined. In view of the above. a third monolithic double reflector was inserted upstream of the high-resolution elements whose sole purpose was to intercept the bulk of the thermal load and prevent it from disturbing the thermal equilibrium of the succeeding stages. It does not need to provide resolution. but it should be efficient. A silicon (1.1.1) monolith was used. Its tolerance of therma! loacs is muct greater that that of the (10.6.4) device. and on the scale of mil-

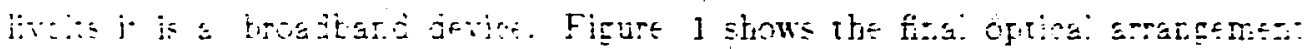

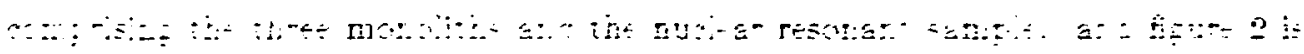

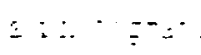

2.3. Mechanical design

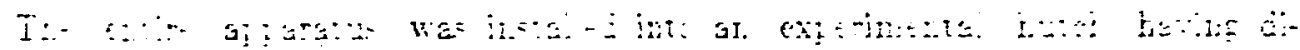

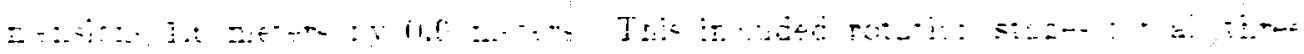

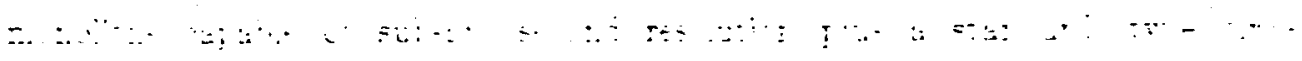

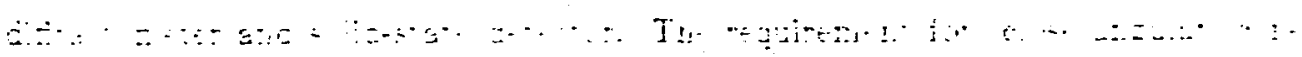


trol over the three optical elements led to a design based on sine bars. Each axis of the high resolution pair consisted of a precision spindle rigidly mounted to the chamber whichi enclosed them. coupled to a lever of length 0.56 meters. The free end of this lever was driven by a non-rotating micrometer bead, which was in turn driven by a stepper motor. The combination was such that one motor step produced a rotation of the spindle of 0.2 arc seconds. The upstream monolith was mounted on a similar arrangement, but with a shorter lever arm ( 0.3 meters). It was surrounded by a lead cave in order to control scatter from the white beam incident on it. A moteable lead beamstop was arranged between the crystals of the high resolution pair. and moveable slits were situated just upstrean; and downstream of them isee figure 1). Thus any spuriously scattered photons were foreed to underge severa! scattering events before exiting the monochromator. The background level was thus rendered very low.

2.4 Alignment

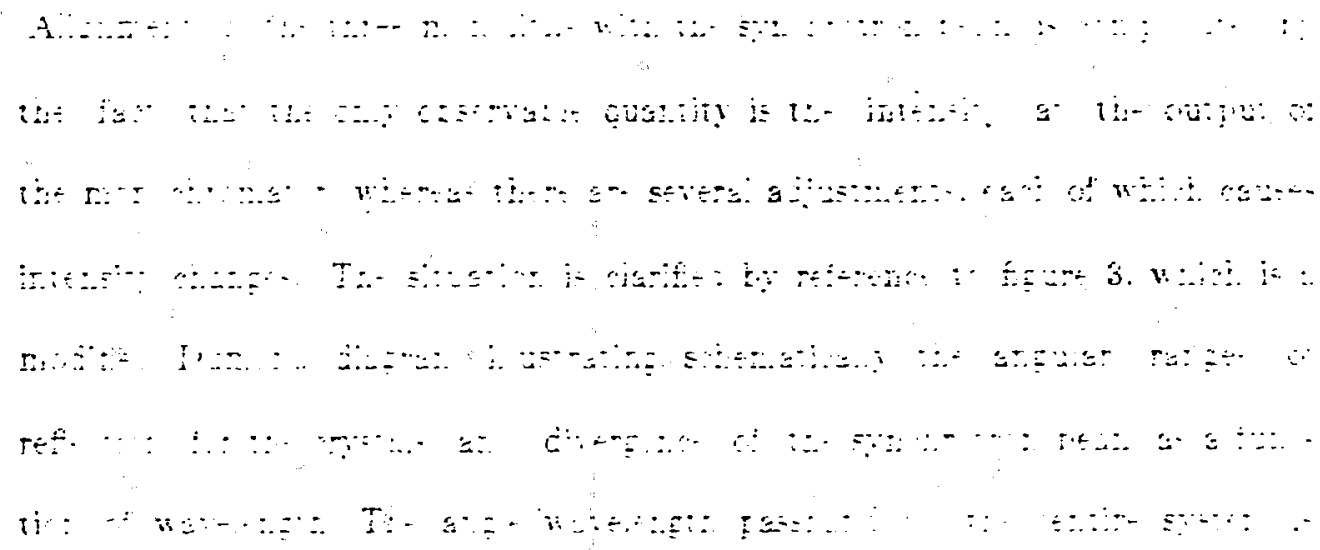


given by the extents of the small parallelogram enclosed by the two (10,6.4) traces. This small area can be made to move anyuhere in the figure by suitable rotations of the two crystals (horizontal translation of the traces in the figure). If one of the $(10,6,4)$ crystals is rotated. the rectangle traverses the $(1,1,1)$ reflectivity range. If the synchrotron beam intensity is uniform (in angle) over this range. then such a scan should map out the silicon (1,1,1) Darwin-Prins curve. Figure 4 shows the result of just such a measurement. The theoretical width of the reflectivity curre is 3.5 arc seconds, whereas the width of the curve in fgure 4 is around 7 arc seconds. Howerer. simple geometric arguments based on figure 3 can show that rotation of one crystal by an angle $i$ causes the center of the parallelogram to move in angle by $u / 2$. as observed.

The most direc algorithw for adjusting the monochramator is as follows. First

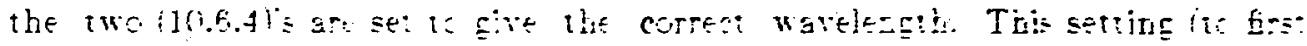

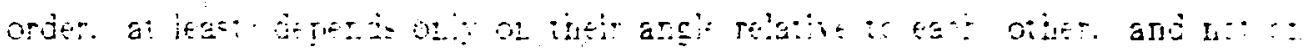

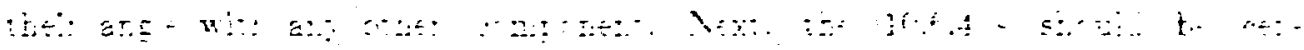

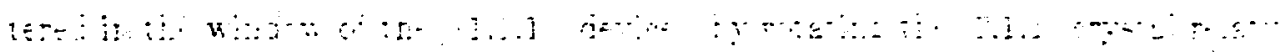

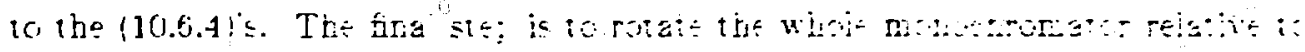

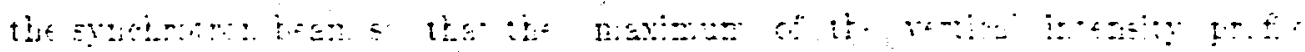

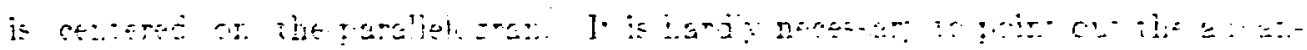

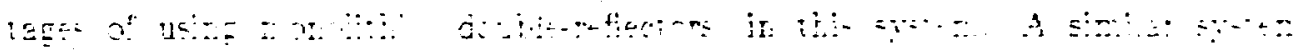

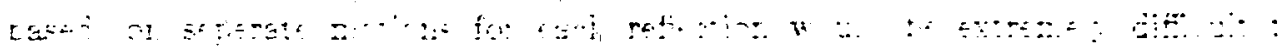
$a !:=1$. 


\section{Wavelength calibration}

Since the resolution of this monochromator is very high, it becomes important to devise a method of calibration of the wavelength scale to a level similar to its resolution. Unfortunately, very few physical effects in the $x$-ray regime are sufficiently sharp or well-determined to justify their use as a calibration point. The Mössbauer line itself is such a feature, and would make an admiratle wavelength standard: significantly better than the current, $x$-ray wavelength standard (as was pointed out by Bearden [9]). However, examination of the literature reveals a range of values quoted for the energy of this line. Bearden $19 ;$;eported measurements made using calcite and quart 2 crystals in a doubie-crysta! spectrometer. The two values ottained differed by 0.2 el. a factor of farty times the resolution of our monochromator! Gerdau et al [ 3 : quote a valut uhich is 0.5 e $\mathcal{H}^{-}$different from the mean of Bearden's values.

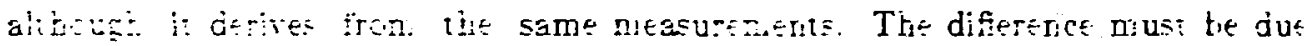

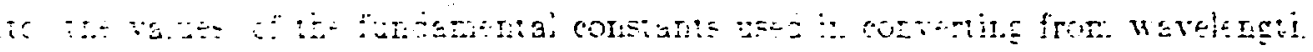

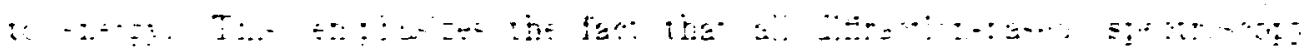

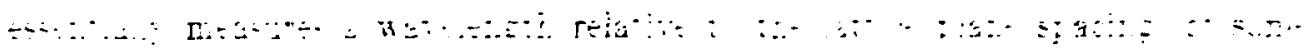

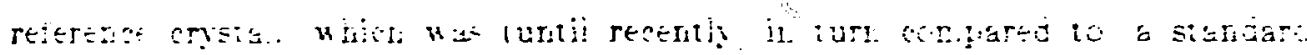
we:

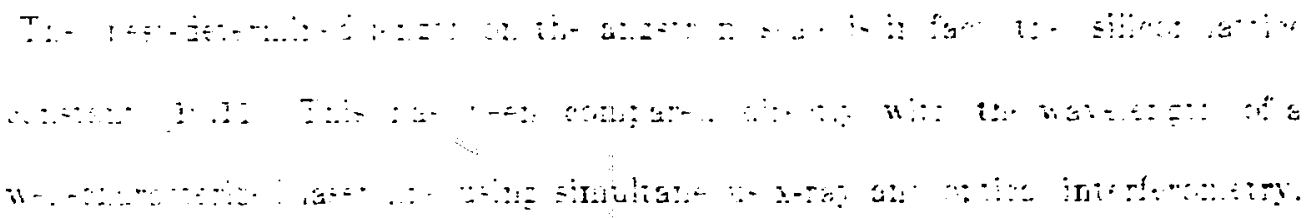


thus breaking the circular relationship described above. It would appear sensible to use this fact in calibration. The output of our monochromator was passed to a two-circle diffractometer upon which was normally mounted our nuclear resonant sample. This diffractometer (a Huber 424) was said to have an absolute accuracy of less than 30 arc seconds. It was fitted with gear reducers and stepping motors which gave it a step size of 0.9 arc seconds. Our experience indicated that its reproducibility is much better than its accuracy, around \pm 1 arc second. The principle purpose of a warelength determination using this instrument would therefore be as an internal calibration. For the purposes of this calibration. the sample was replaced by a $0.5 \mathrm{~m} . \mathrm{m}$. thick silicon plate. This plate was cut in such a way that the $(\overline{10} .6 .4)$ planes were parallel to its major face. and the (1.1.1) planes were perfendicular to that face (see figure 5). The Bragg angle for the (10.6.4) reflection at $14.413 \mathrm{ket}$ is 77.5 degrees. For the (1.1.1) reflection it is 7.9 degress. Thus the crisial orientation for the

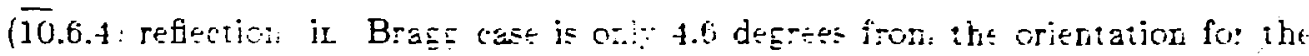

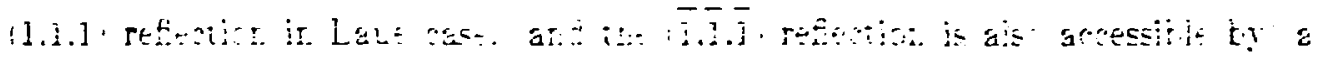

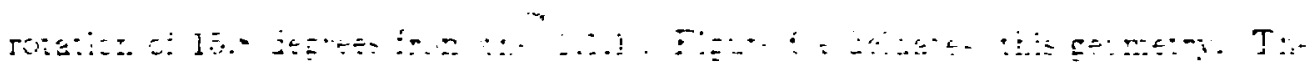

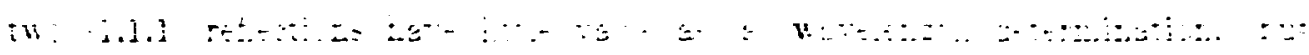

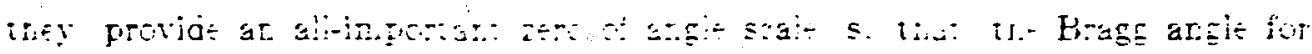

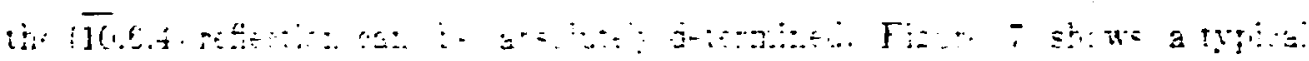

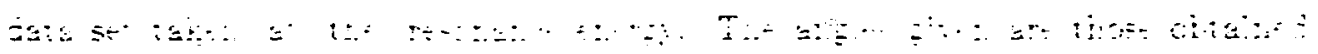

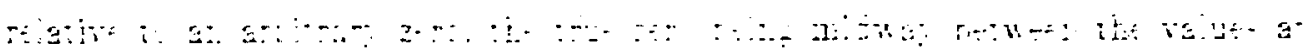

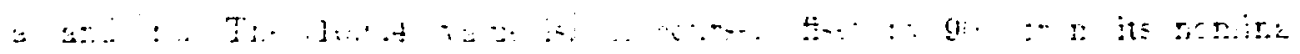

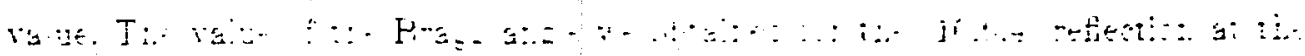


Mössbauer resonance was $77.53350^{\circ}$, which corresponds to wavelength of $0.860262 \AA$. Various systematic errors were unaccounted for in these initial measurements. and so this value does not constitute an absolute determination of the wavelength of the Mössbauer line. Future measurements will take proper account of these errors and an improved value for the wavelength will result. Since the present level of knowledge of the line energy is around $0.2 \mathrm{eV}$, a search was still necessary initially to locate the exact resonance energy. Once it had been found. however. the above calibration scheme enabled us to return to the correct energy within a very short time.

\section{Measurements}

As described above. the absolute value of the resonance wavelength is ratber ponriy defined compared to the monochromator passband. It is therefort

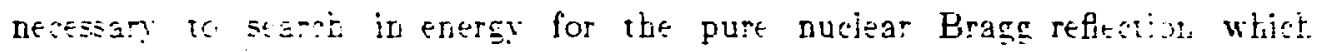

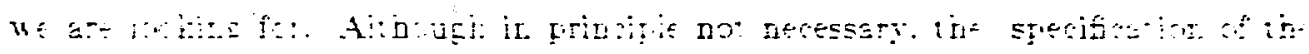

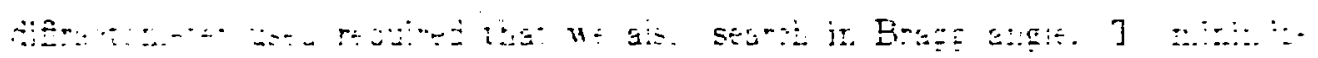

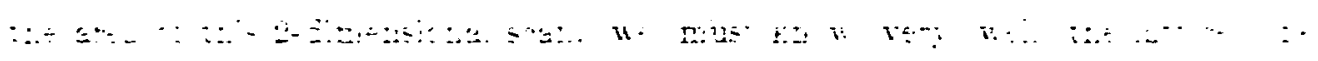

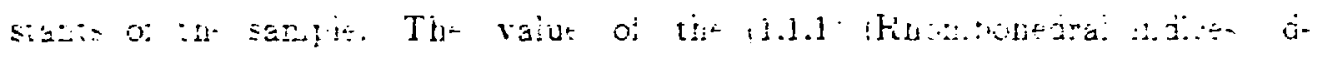

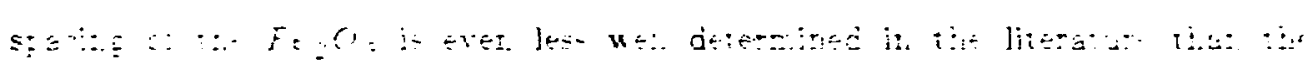

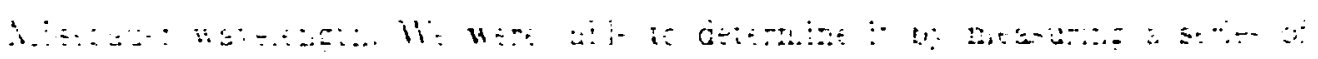

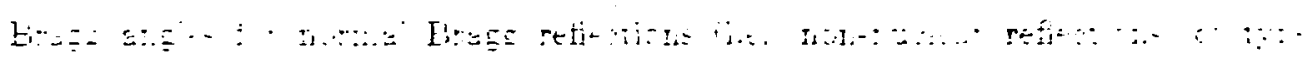

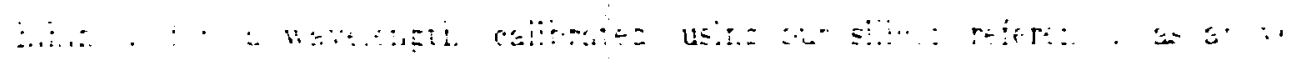

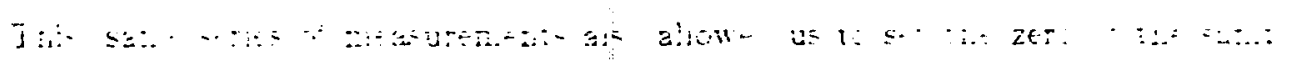


Bragg-angle scale. This is different from the zero in the wavelength calibration experiment since the crystal did not have a well-determined orientation relationship with the goniometer mount. The search for this peak $.005 \mathrm{~V}^{\circ}$ and 3 arc seconds vide in a parameter space $1 \mathrm{eV}$ and 20 arc seconds wide took a considerable time. Once found. the resonant sample was removed and the wavelength determined as described above. Subsequent searches from this calibration point usually took around thirty minutes.

Figure 8 shows two rocking curves of the $\mathrm{Fe}_{2} \mathrm{O}_{3}$ crastal at the resonance energy. The upper curve is a normal Bragg peak (the allowed (6.6.6) reflection). whereas the lower is from the pure nuclear (7.7.7) reflection. The notable feature is the reflection widths, in that they are essentialy the same. This implies that the width is characteristic of the strains in the crystal sample. Bycontrast. if we scan the samit two refiections in wavelength. as in figure 9 (i.e. ai fixed crysia! angle white changing waveieng:t we sef the dramaits

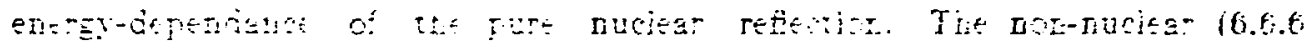

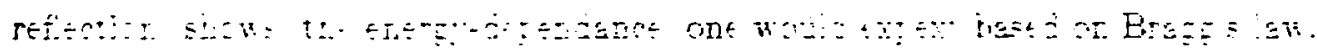

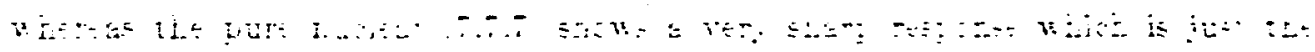

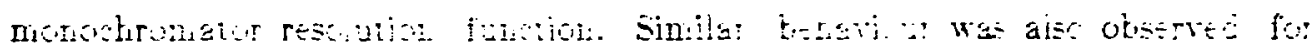

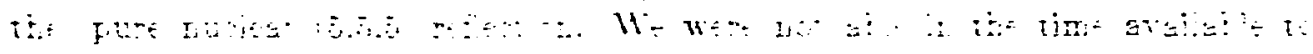

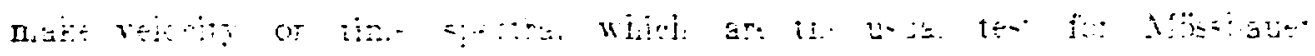

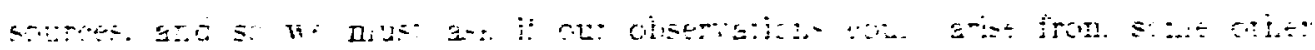

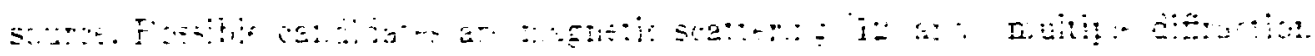

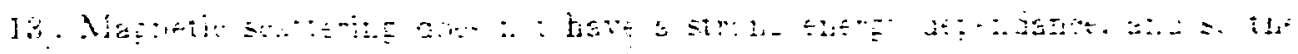


data of figure 8 removes that possibility. Multiple diffraction is more difficult to discount, but several facts suggest strongly that our observation is nol due to this phenomenon. First, any diffraction phenomenon in our crystal will be smeared to some extent by its grown-in strains, and this would be reflected in similar ways in both angular and energy scans. Comparison of figures 8 and 9 show the marked difference between angle and energy dependance of the pure nuclear reflection. Secondly, the occurrance of an accidental multiple diffraction point at exactly the same energy (to within a few parts in $10^{5}$ ) as the nuclear resonance is highly unlikely, and the added coincidence of a similar peak observed at the pure nuclear (5.5.5) reflection is not reasonable.

There are other features of the data which are interesting from the point of visu of resonant nuclear diffaction. but the reader is referred to reference 114 : for a discussin of ten.

5. Summary

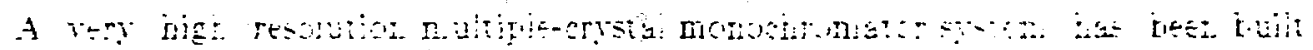

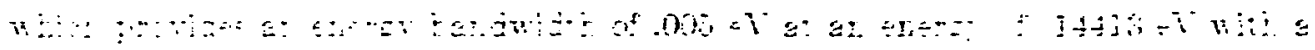

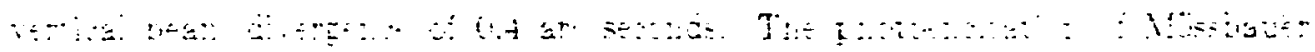

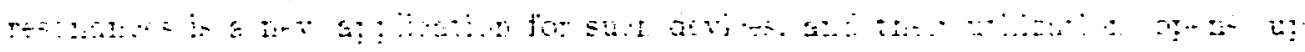

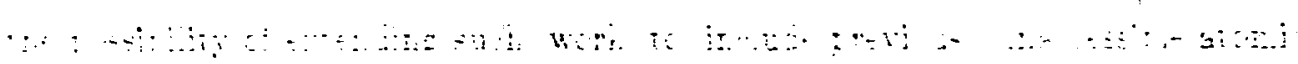

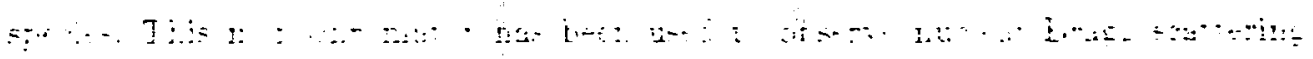


from a single pure nuclear reflection with a signal to noise ratio of 100:1. It is also intriguing to consider the application of this monochromator system. coupled with a similar dispersive double-crystal analyzer to inelastic $\mathrm{x}$-ray scattering. Such a system would be ideal for probing inelastic scattering in the region of very small momentum transfer as well as the study of inelastic features which are sharp in momentum (i.e. having widths similar to Bragg reflection widths).

\section{Acknowledgments}

We wish to acknowledge the contribution of J. P. Remeika and A. S. Cooper of ATdT Bell laboratories who grew the remarkably good crystal of isotopically enriched ${ }^{5-} \mathrm{Fe}_{2} \mathrm{O}_{3}$. J. R. Grover and P. E. Haustein have also pade valuable contritutions to the ruclear Bragg diffraction program at B.N.L. We are also grateful to R. V. Pound. wì gave much encouragement and useful discussici.

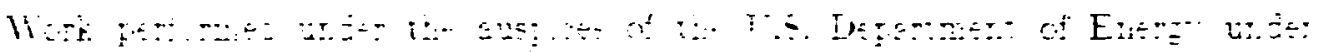

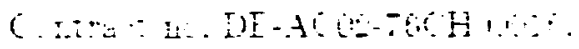




\section{References}

1. R. L. Mössbauer, Z. Physik 151 (1958) 124: Naturwissenschaften 45 (1958) 538: Z. Nat urforsc. $14 a(1959) 211$.

2. S. L. Ruby. J. Phys (Paris). Colloq. 35 (1974) C6-209

3. (a) E. Gerdau, R. Ruffer, H. Winkler. W. Tolksdorf, C. P. Klages \& J. P. Hannon. Phys. Rer. Lett. 54 (1985) 835. (b) E. Gerdau. R. Ruffer, R. Hollatz.J. P. Hainon. Phys. Rev. Lett. 57 (1986) 1141.

4. 11. Graeff and G. Materlik. Nucl. Instrum. and Meth. 195 (1982) 97.

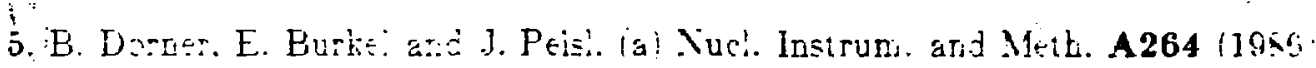

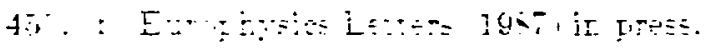

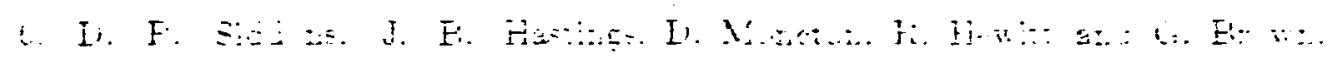

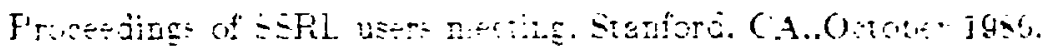

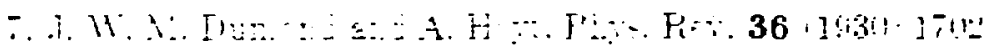

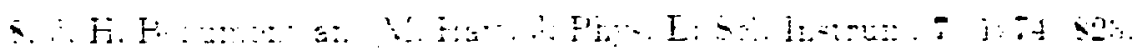


9. J. A. Bearden, Phys. Rev. B137 (1965) 455.

10. R. D. Deslattes and A. Henins. Phys. Rev. Lett. 31 (1978) 972.

11. P. Becker, K. Dorenwendt. G. Ebeling. R. Lauer. W' Lucas, R. Probst, H- J.

Rademacher, G. Reim, P. Seyfried and H. Siegert. Phys. Rev. Lett. 46 (1981) 1540.

12. M. Brunel and F. de Bergerin. Acta Cryst. A37 (1981) 324.

13. R. 11. James. The Optical Principles of the Diffraction of X-rays. Cornell University Press. Ithaca. X'Tw York. (1965) $2(0$.

14. G. Faigel. D. P. Sidjons. J. B. Hastings. P. E. Hausteir. J. P. Grover. J. P.

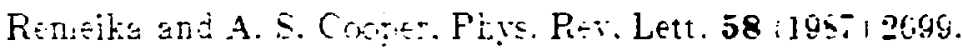


Figure captions.

1. Disposition of monochromator components and nuclear resonant sample crystal, showing beam trajectory.

2. (a) Photograph of silicon monoliths used in the monochromator. (b) Pho tograph of perfect single crystal of ${ }^{57} \mathrm{Fe}_{2} \mathrm{O}_{3}$.

3. Modified DuMond diagram showing angular ranges of reflection of the crystals and divergence of the synchrotron radiation beam as a function of wavelength.

4. Rocking curve produced by rotating one of the (10.6.4 crystals wit: al! the

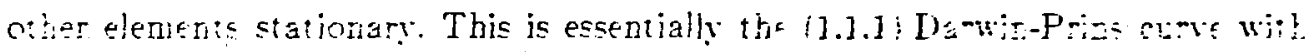

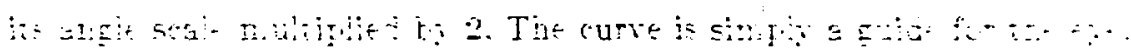

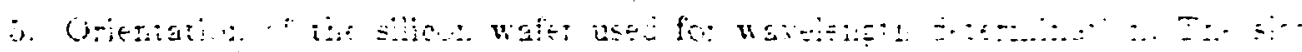

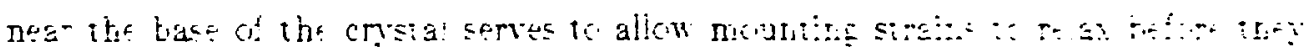

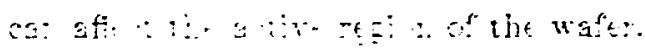

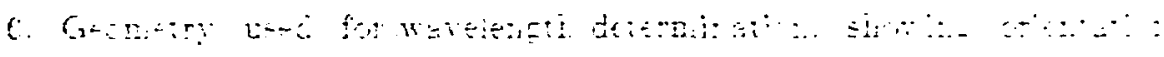

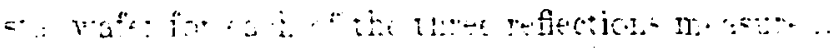


7. Intensity profiles for the three reflections used for wavelength determination. The data points are joined by straight lines to guide the eye.

8. Rocking curves of the normal $(6,6,6)$ Bragg peak (i.e. due to electronic scattering) and of the $(7,7,7)$ pure Nuclear Bragg reflection, on resonance. The two curves have essentially the same shape which is dominated by the sample crystal perfection.

9. Energy scans at constant Bragg angle for the (6.6.6) and (7.7.j) reflections. The energy widh of the (6.6.6) reflects the crystal perfection. and is consistant with Bragg's law. whereas the energy width of the (7.7.7) is just the passband of the monochromator. and verifies the pure nuclear nature of the reflection. 


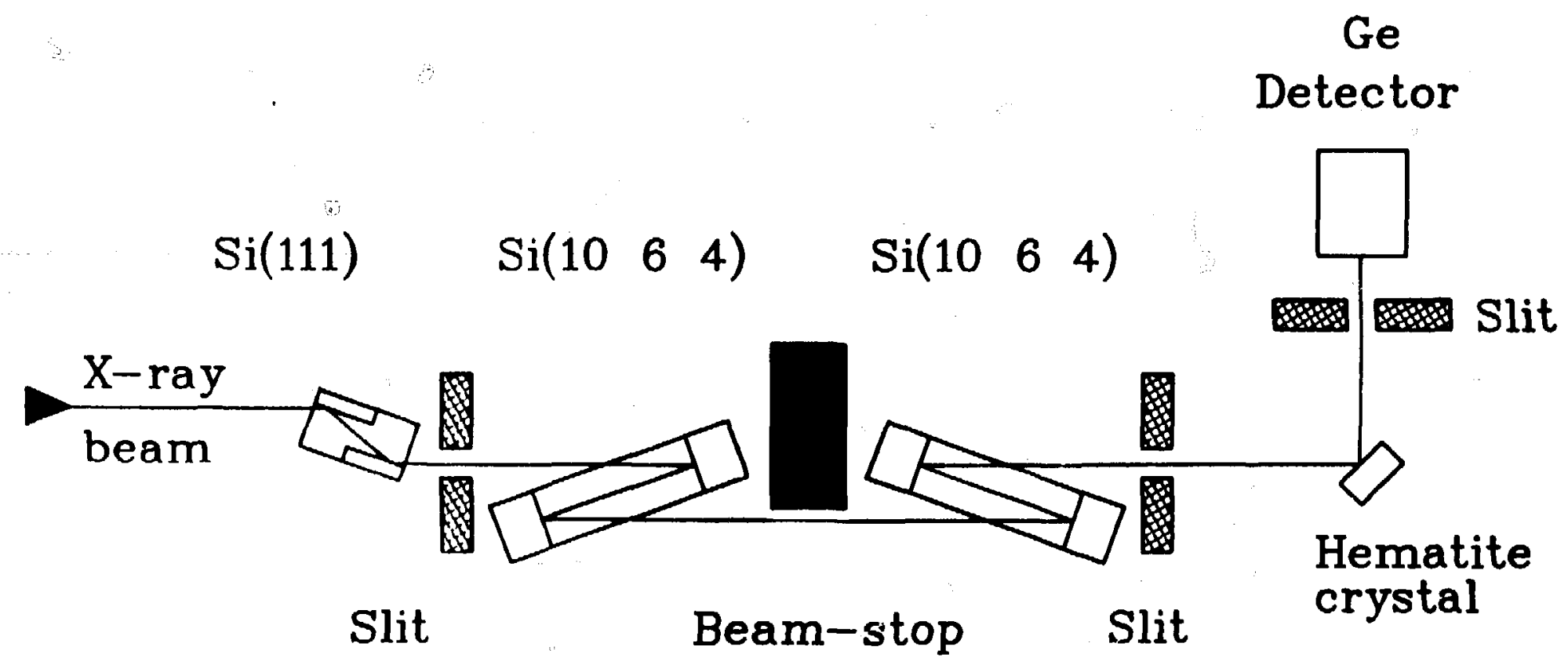

$f_{i n} 1$. 

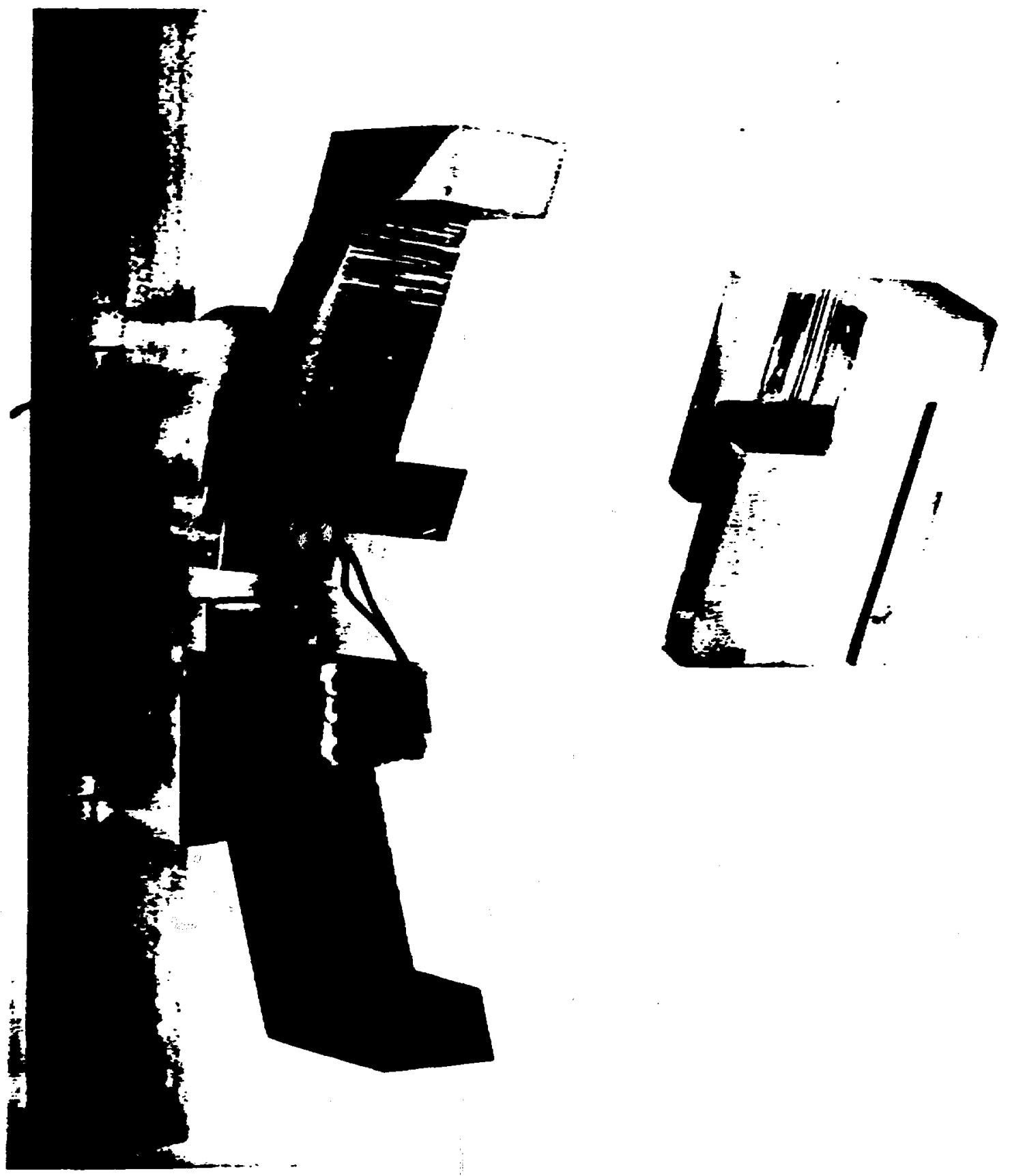

3

$4^{5}$ 


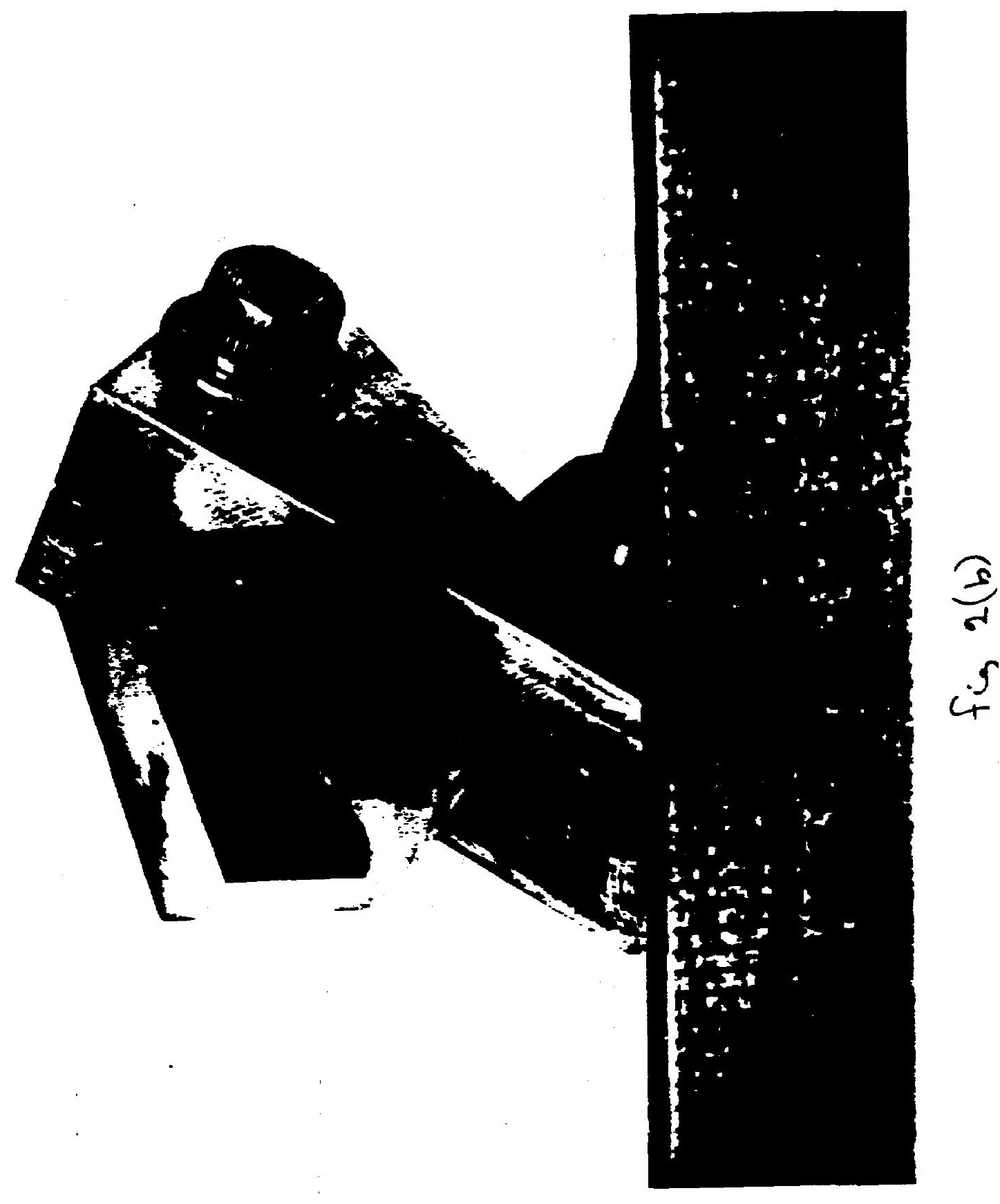




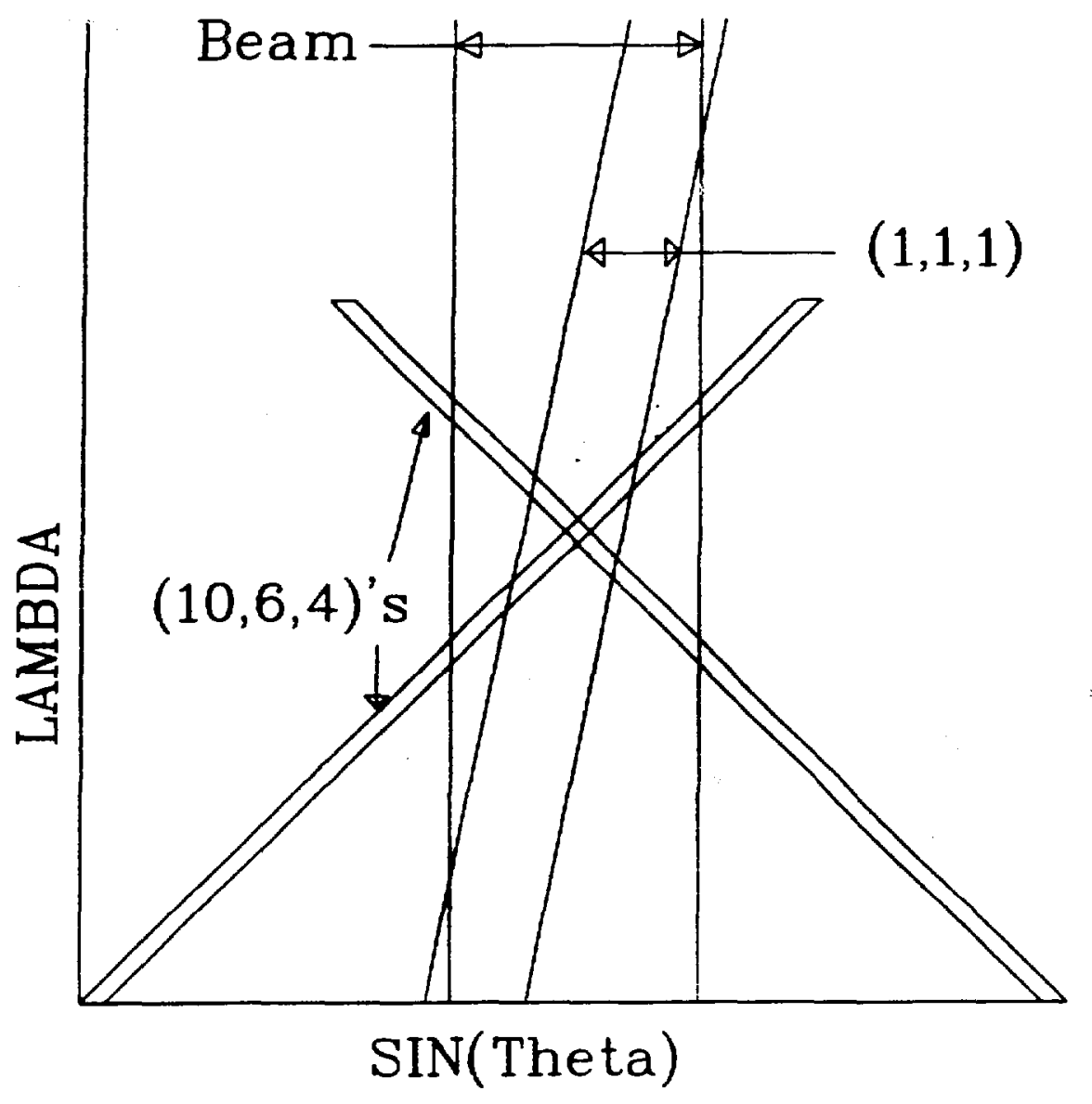

fig 3. 


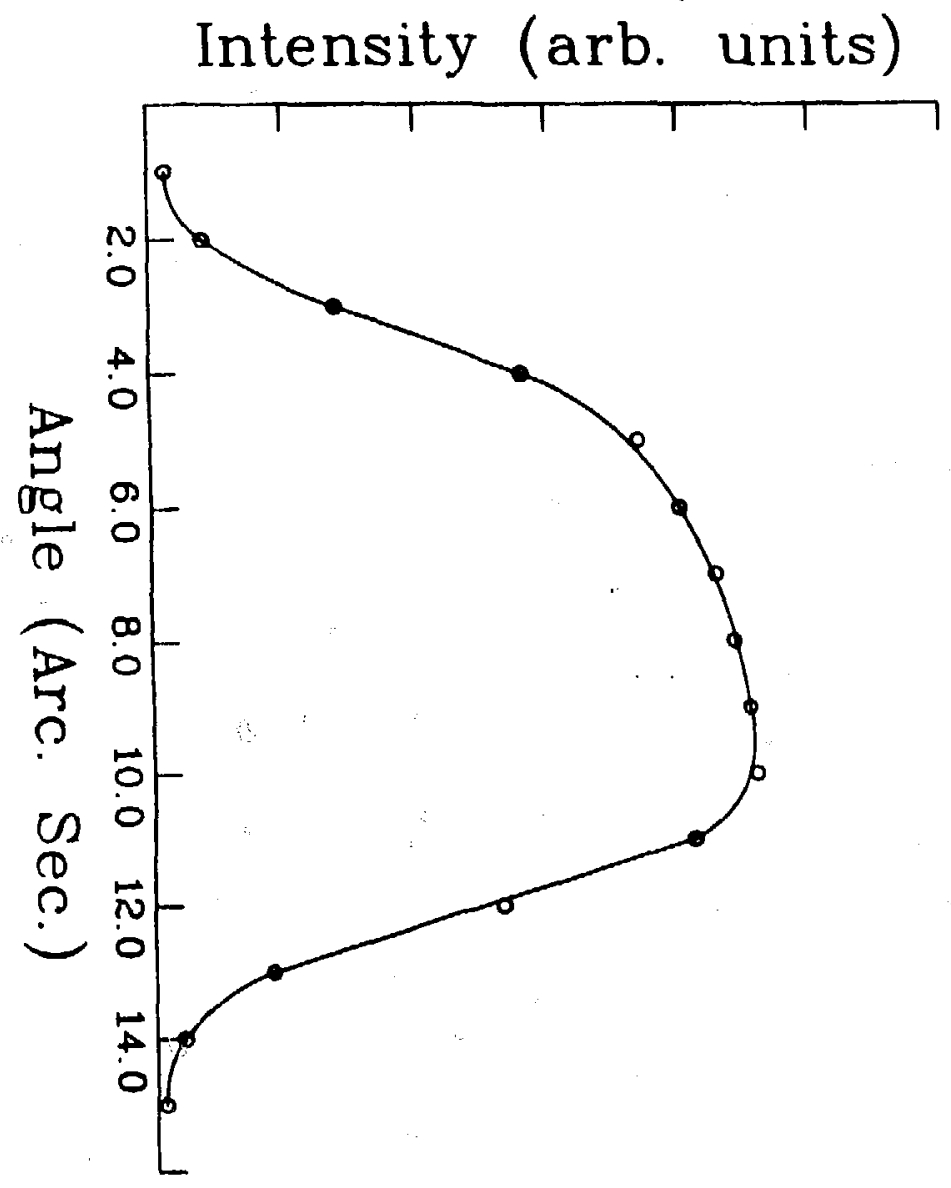




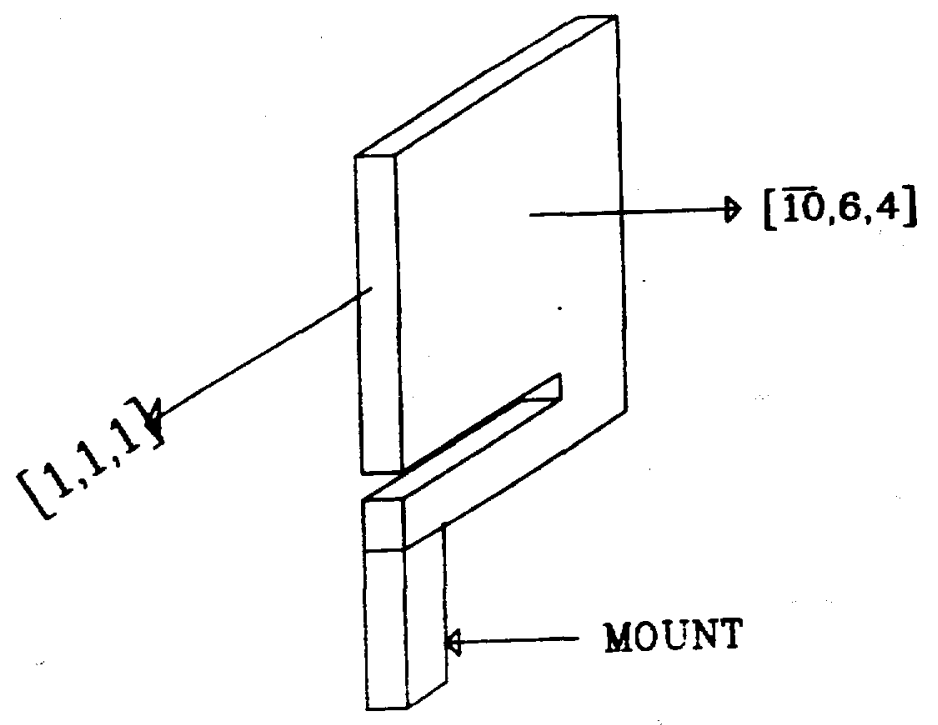

fig 5 

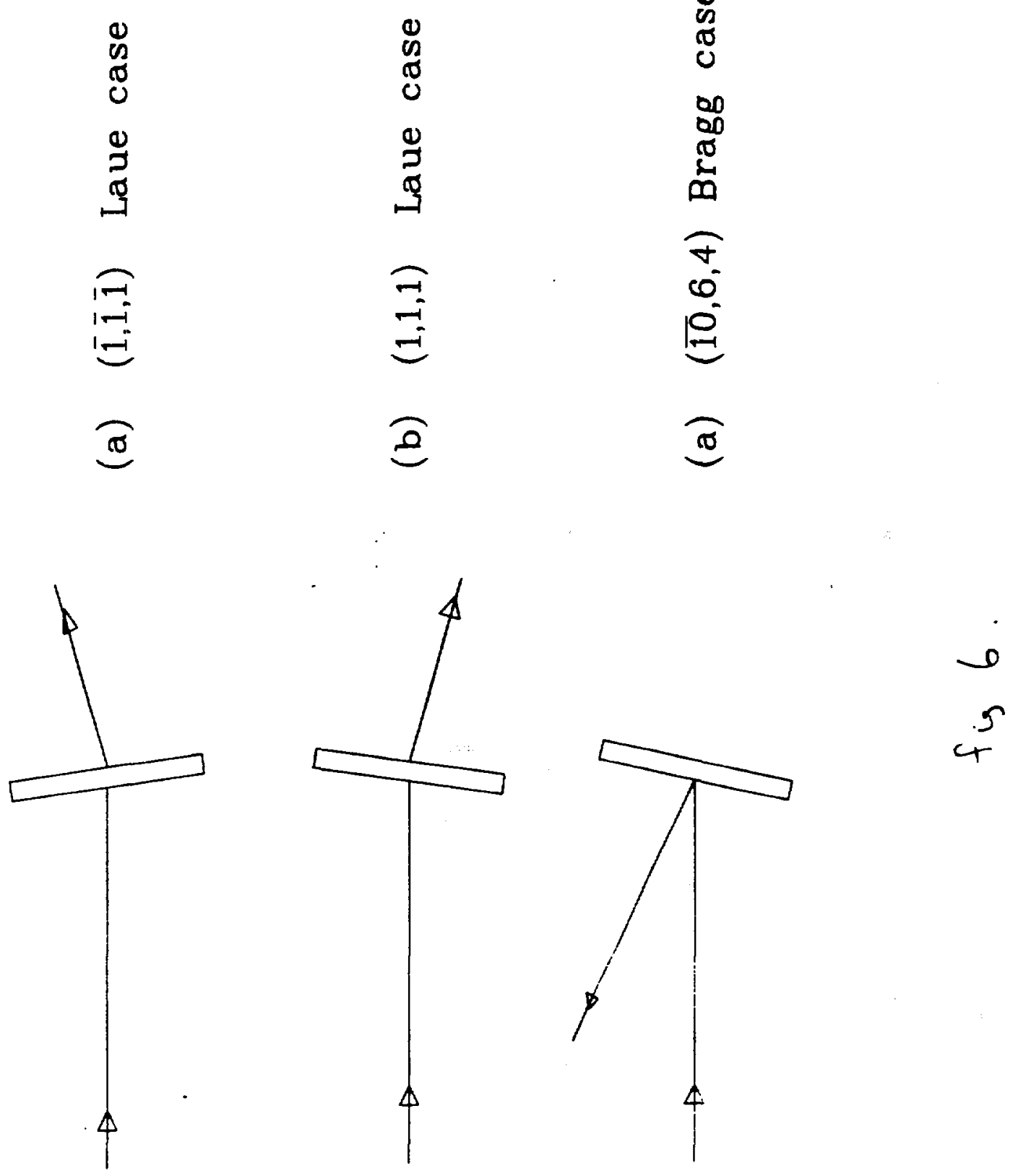


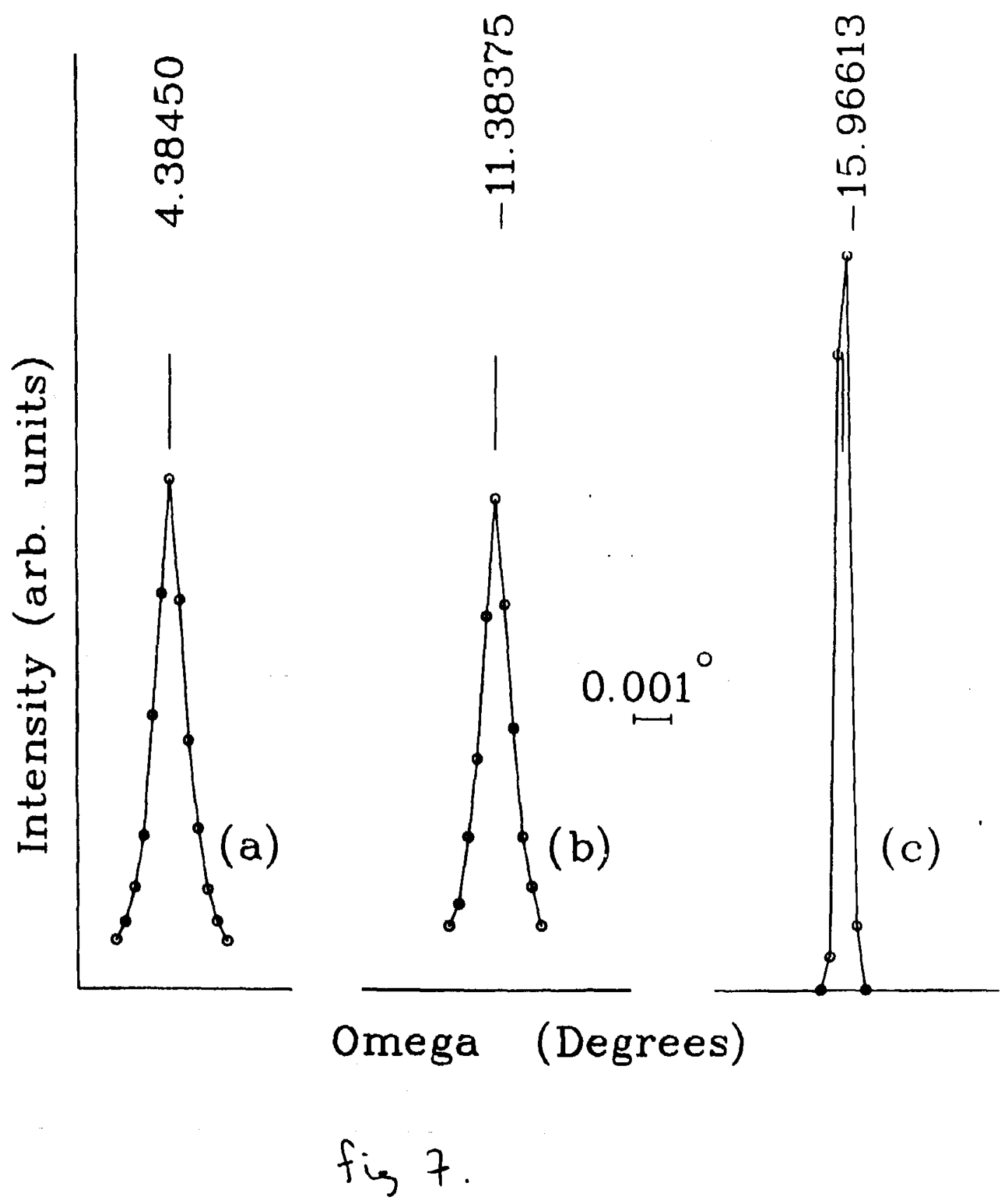




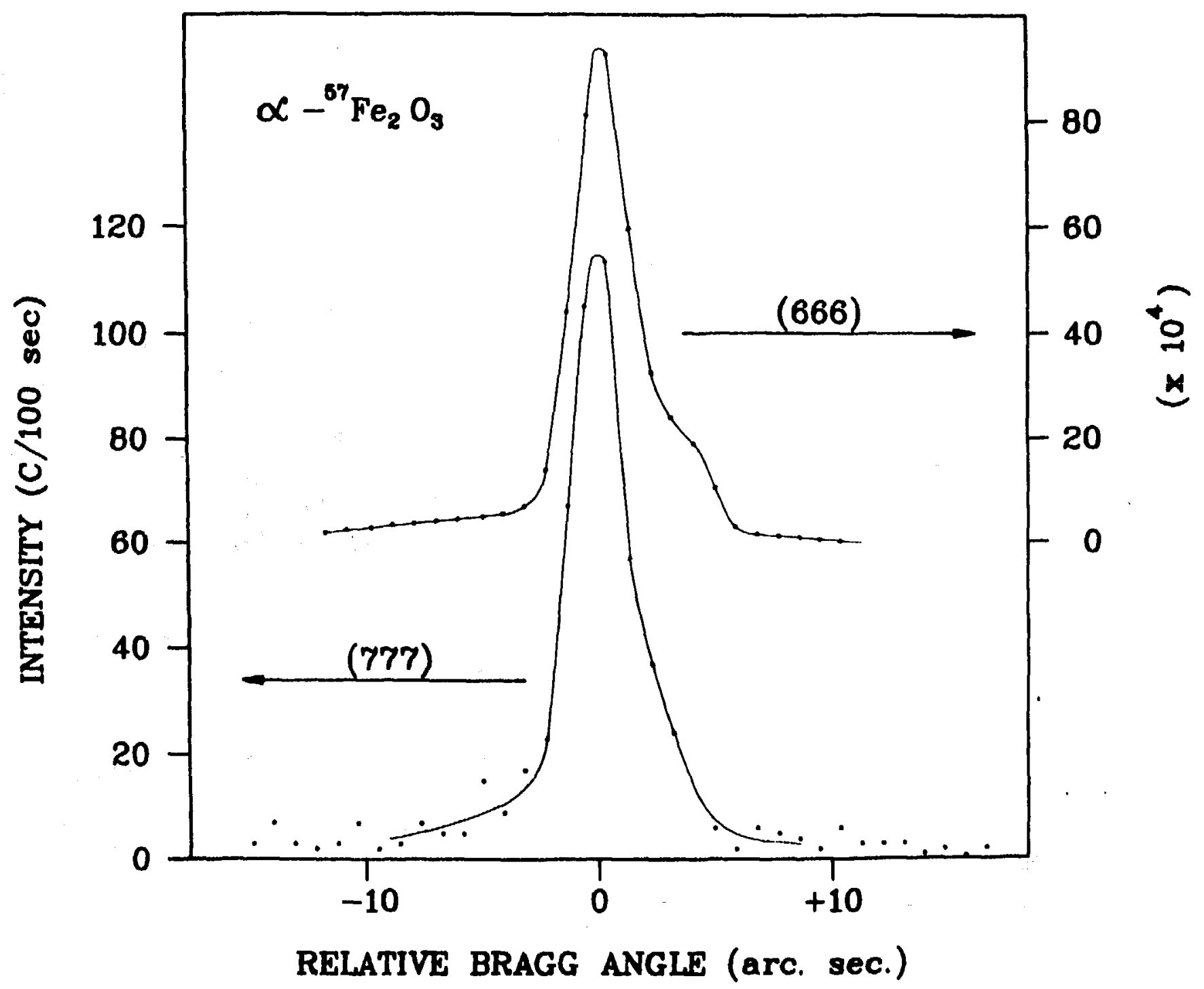

fies 8 


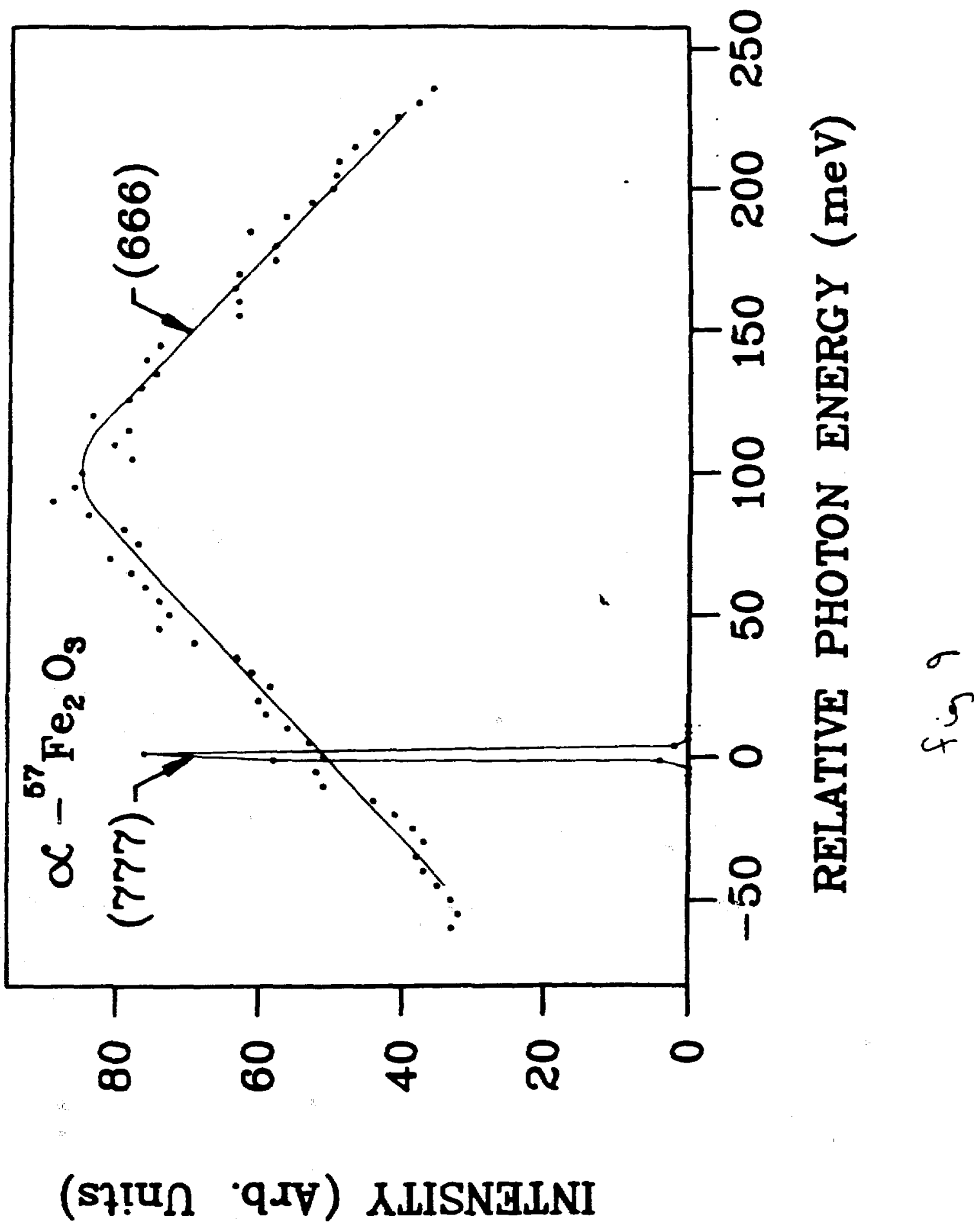

